\title{
Canadian national COVID-19 genomics surveillance priorities for existing and emerging variants of concern
}

\author{
on behalf of the Genome Canada Canadian COVID-19 Genomics Network (CanCOGeN) and \\ the Canadian Public Health Laboratory Network CanCOGeN Working Group
}

\begin{abstract}
Suggested citation: Genome Canada Canadian COVID-19 Genomics Network (CanCOGeN) and the Canadian Public Health Laboratory Network CanCOGeN Working Group. Canadian National COVID-19 genomics surveillance priorities for existing and emerging variants of concern. Can Commun Dis Rep 2021;47(3):139-41. https://doi.org/10.14745/ccdr.v47i03a03

Keywords: Genomic surveillance, genomic sequencing, COVID-19, variants of concern, SARS-CoV-2, Canada, public health, surveillance
\end{abstract}

\section{Introduction}

The Canadian COVID-19 Genomics Network (CanCOGeN) (COVID-19, coronavirus disease 2019) is performing genomic surveillance of circulating severe acute respiratory syndrome coronavirus 2 (SARS-CoV-2) in Canada to track its spread, monitor for variants of concern (VOCs) that might impact transmissibility or disease severity, assist in outbreak investigations and assess the impact of public health interventions. Recent reports of emerging VOCs with enhanced transmissibility have been reported in the United Kingdom (UK) and South Africa (SA). The potential for rapid spread of these variants affirms the need for ongoing and enhanced genomic surveillance in Canada and worldwide. In this guidance document, we set out the national priorities for genomic surveillance, including targeted surveillance of existing and emerging VOCs.

\section{Targeted genomic surveillance of variants of concern (VOC-202012/01) and N501Y.V2}

The COVID-19 variant, VOC-202012/01, was first detected in October 2020 in the UK. Its presence was correlated with increased transmissibility in the UK and has been reported in other countries, including Canada. Another newly emerged SA VOC, designated N501Y.V2, similarly correlates with increased transmissibility. As of January 7, 2021, the N501Y. V2 variant has not yet been detected in Canada. Both variants are defined by an "N501Y" mutation in the SARS-CoV-2 spike protein's receptor-binding domain. There is currently no evidence that either VOC results in increased severity or impacts vaccine efficacy. The CanCOGeN has identified both VOCs as priorities for targeted genomic surveillance.
This work is licensed under a Creative Commons Attribution 4.0 Internationa License.

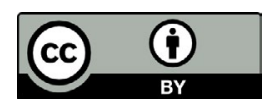

Correspondence: gary.vandomselaar@canada.ca
- Prospective targeted genomic surveillance (Priority: highest)

This includes all international travellers, including from the United States, and close contacts, from the present until further notice.

- Retrospective targeted genomic surveillance (Priority: medium)

This includes all international travellers, including from the United States, and close contacts, from September 1, 2020, to the present.

- Multi-target COVID-19 RT-PCR tests with S-gene target dropouts (Priority: high)

The UK VOC-202012/01 variant can test negative for the S-gene target but positive for other targets using the three-target assay (N, ORF1ab, S) from Thermo Fisher (TaqPath). Multi-target reverse transcription polymerase chain reaction (RT-PCR) assays that include a S-gene target that are affected by the deletions present in the variant can be used as a signal for follow up confirmatory genome sequencing. 


\section{Genomic surveillance of emerging variants of concern}

- Suspected reinfection (Priority: medium) We define suspected reinfection as clinical recurrence of symptoms compatible with COVID-19, accompanied by positive polymerase chain reaction (PCR) (Ct less than 35), more than 90 days after the onset of the primary infection, supported by close contact exposure or outbreak settings, and no evidence of another cause of infection (1).

Reinfection indicates possible infection by immune-escape variants.

- Severe acute COVID-19 in individuals younger than 50 years old without significant comorbidities (Priority: medium)

Disproportionately severe disease in individuals who are otherwise healthy may indicate a change in pathogen virulence resulting in a more florid clinical phenotype, and is thus relevant for surveillance and potentially for patient management.

- Vaccinated individuals with subsequent laboratoryconfirmed SARS-CoV-2 infection (Priority: medium) Although there is a limited number of vaccinated individuals at this time, that number is expected to grow. It is anticipated that with the rollout of vaccines there will be a need to monitor for and characterize potential vaccineescape variants. This likely would require simultaneous monitoring for immune correlates of vaccine response, assessment of seroprotection and systematic genomic testing of post-vaccine infections to monitor for vaccineescape mutants.

- Known or suspected super spreading events (Priority: medium)

Given the proposed potential for increased transmissibility of VOC-202012/01 and N501Y.V2, and the N501Y mutation that they share, sequencing multiple samples from a known or suspected superspreading event may identify such mutations. Sampling the index cases in outbreaks may provide the highest yield.

DEFINITION: A superspreading event is a type of outbreak where there is additional epidemiological and/or genomic evidence of one person with overdispersed transmission of COVID-19, (i.e. directly transmitting to at least five non-household individuals). The statistical concept of overdispersion refers to the few individuals disproportionately and directly infecting a large number of secondary cases relative to the "average" infectious individual, whose infectiousness may be represented by RO, which is estimated at 2.0 for COVID-19 (2).

EXCLUSIONS: This definition excludes large or propagated outbreaks with no evidence of overdispersion.
- Geographic sampling in subregions with a pronounced increase in the case notification rate (Priority: high) A rapid increase in the case positivity rate in a geographic region may indicate either the possible presence of the UK and/or SA variants potentially contributing to increased cases/positivity (given the proposed potential for increased transmissibility of the UK and SA VOCs, and the N501Y mutation), or represent the context within which VOCs with increased transmission potential can take off. Public health authorities could perform geographic sampling in subregions where the positivity rate or per-capita rates or estimated reproductive rate is of higher magnitude and especially if increasing faster (or the doubling time is shorter and/or decreasing) as compared with the provincial average. Ideally, identifying the subregions for sampling would exclude cases in congregate settings (e.g. long-term care homes). Such subregions may overlap with the density of physical contact networks (e.g. greater household density and/or occupational exposures). These could be at the sub-provincial level (e.g. public health unit, city, etc.) or sub-regional level (e.g. neighbourhood).

\section{Other priorities}

- Continued random sampling for routine national genomic surveillance (Priority: high)

The $\mathrm{CanCOGeN}$ sampling guidelines for national priorities include random sampling for routine SARS-CoV-2 genomic surveillance. Routine surveillance is used to monitor existing variants of concern, identify emerging variants of concern, track viral transmission and assess the effectiveness of public health interventions. Random sampling for routine genomic surveillance is ongoing and will continue.

- Continued sampling to investigate SARS-CoV-2 outbreak clusters (Priority: medium)

The CanCOGeN sampling guidelines include strategies to investigate and respond to SARS-CoV-2 outbreak clusters. Sampling for outbreak investigations is ongoing and will continue.

\section{Recommended response}

Individuals with SARS-CoV-2 infections that are compatible with the above groupings may signal an existing or new variant of concern. As a result, beyond the existing suite of public health measures in place, it is recommended that obtaining samples that enable downstream sequencing is a high priority. Following collection, specimens from such cases should be forwarded to the public health lab in their region to be sequenced in a timely manner to identify cases of the new variant. If the UK or SA variants are detected, enhanced genomic surveillance should be conducted in the community/region/event. 
Competing interests

None.

\section{Acknowledgements}

The Genome Canada input is derived from the Canadian COVID-19 Genomic Network (CanCOGeN) Working Group and the Canadian Public Health Laboratory Network (CPHLN) input is derived from the CPHLN CanCOGeN Working Group and. We would like to thank members of the CPHLN Secretariat, including MJ Lacombe for coordinating the document synthesis.

\section{Funding}

None.

\section{References}

1. Yahav D, Yelin D, Eckerle I, Eberhardt CS, Want J, Cao B, Kaiser L. Definitions for COVID-19 reinfection, relapse and PCR re-positivity. Clin Microbiol Infect 2021;27(1):315-8. DOI

2. Liu Y, Gayle AA, Wilder-Smith A, Rocklöv J. The reproductive number of COVID-19 is higher compared to SARS coronavirus. J. Travel Med. 2020;27(2):taaa021. DOI 